\title{
Brain Death in Children: Incidence, Donation Rates, and the Occurrence of Central Diabetes Insipidus
}

\author{
Nazik Yener*, Muhammed Şükrü Paksu, Özlem Köksoy \\ Ondokuz Mayıs University School of Medicine, Division of Pediatric Critical Care, Samsun, Turkey
}

\begin{abstract}
Introduction: Brain death is currently defined as the loss of full brain function including the brainstem. The diagnosis and its subsequent management in the pediatric population are still controversial. The aim of this study was to define the demographic characteristics, clinical features and outcomes of patients with brain death and determine the incidence of brain death, donation rates and occurrence of central diabetes insipidus accompanying brain death in children.
\end{abstract}

Methods: This retrospective study was conducted at a twelve-bed tertiary-care combined medical and surgical pediatric intensive care unit of the Ondokuz Mayıs University Medical School, Samsun, Turkey. In 37 of 341 deaths (10.8\%), a diagnosis of brain death was identified. The primary insult causing brain death was post-cardiorespiratory arrest in 8 (21.6\%), head trauma in 8 (21.6\%), and drowning in $4(18.9 \%)$. In all patients, transcranial Doppler ultrasound was utilised as an ancillary test and test was repeated until it was consistent with brain death.

Results: In 33 (89\%) patients, central diabetes insipidus was determined at or near the time brain death was confirmed. The four patients not diagnosed with CDI had acute renal failure, and renal replacement treatment was carried out. The consent rate for organ donation was $18.9 \%$, and $16.7 \%$ of potential donors proceeded to actual donation.

Conclusion: In the current study the consent rate for organ donation is relatively low compared to the rest of the world. The prevalence of central diabetes insipidus in this pedaitric brain death population is higher than reports in the literature, and acute renal failure accounted for the lack of central diabetes insipidus in four patients with brain death. Further studies are needed to explain normouria in brain-dead patients.

Keywors: brain death, children, diabetes insipidus, cerebral blood flow, organ donation

Received: 31 October 2017 / Accepted: 26 January 2018

\section{INTRODUCTION}

Brain death (BD) is currently defined as the loss of full brain function including the brainstem, characterised by deep coma, apnea and lack of supraspinal reflexes. A patient determined to be BD is accepted nearly worldwide as being clinically and legally dead [1]. Although Turkey, in 1979, was one of the first countries to establish a legal framework for organ transplantation, and also has the facilities capable of performing the necessary procedures, the number of $\mathrm{BD}$ declarations and the consent rate for organ donation is relatively low compared to the rest of the world [2]. Children on an organ transplant waiting list, die as a consequence of the shortage of organs available to meet the demand for transplantation. Therefore, the diagnosis of $\mathrm{BD}$ and the care of the donor organs are vital.

Central diabetes insipidus (CDI) due to antidiuretic hormone deficiency is a well-described complication of $\mathrm{BD}$ in both children and adults and is characterised by polyuria, hypernatremia, and hyperosmolar dehydration [3-5]. It is not surprising that CDI develops in patients who are $\mathrm{BD}$. On the contrary, it is puz- 
zling that CDI does not always occur following BD. The incidence of CDI accompanying BD in adults and children varies. In a recent review of 32 studies, of the 1878 patients with $\mathrm{BD}$, ranging in age from 2 months to 89 years, 925 (49\%) were reported to have CDI [6]. At least some of thenormouric patients in these studies had some preserved brain function through hypothalamic osmoregulation. Although the term "brain death" is sometimes used to mean irreversible cessation of all functions of the entire brain, this implies that continued hypothalamic function in $\mathrm{BD}$ is a contradiction in terms.

Even with clear BD criteria in place for over three decades, diagnosis and subsequent management of the pediatric population are still controversial and cause anxiety in the medical community all over the world.

The aim of this study was to define the demographic characteristics, clinical features and outcomes of patients with $\mathrm{BD}$ and determine the incidence of CDI accompanying $\mathrm{BD}$ in children.

\section{MATERIAL AND METHODS}

Approval for the study was granted by the Ethics Committee of the Ondokuz Mayis University. This retrospective study was conducted at 12-bed tertiary-level care combined medical and surgical pediatric intensive care unit (PICU) of the Ondokuz Mayis University Medical School, Samsun, Turkey. The hospital information database systems were used to collect data. All deaths that occurred in PICU over a 5-yr period from 2012 to 2016 were screened. The medical records of pediatric patients, ( $>1$ month and $<18 \mathrm{yr}$ ), who were diagnosed with $\mathrm{BD}$ were evaluated, and retrospective chart reviews were recorded. Data comprising demographics, major cause leading to $\mathrm{BD}$, duration of $\mathrm{BD}$ evaluation and hospitalisation,existence of CDI, and organ donation were collected and documented retrospectively for statistical evaluation. According to Turkish law, the diagnosis of $\mathrm{BD}$ requires the confirmation of lack of clinical functions of the brain, as judged by apnea, brain stem areflexia, and cerebral unresponsiveness, coupled with a known, irreversible cause of coma and an ancillary test for children. Two examinations, including apnea testing, with each examination separated by an observation period, are required. An observation period of twelve hours for infants and children aged 30 days to 18 years, is needed. The final decision regarding $\mathrm{BD}$ must be confirmed by a commit- tee of physicians. Relatives of patients were informed about the declaration of BD by an experienced PICU physician working in the unit. The request for organ donation was made by the organ donation coordinator of the hospital.

CDI has been defined as polyuria (urine output $>$ $4 \mathrm{~mL} / \mathrm{kg} / \mathrm{hr}$ for children $300 \mathrm{~mL} / \mathrm{hr}$ for adult-sized children $\geq 70 \mathrm{~kg}$ ) for at least two consecutive hours, hypernatremia (Serum Na $>145 \mathrm{mmol} / \mathrm{L}$ ), high serum osmolality ( $>300 \mathrm{mOsm} / \mathrm{kg}$ ) and low urine osmolality $(<300 \mathrm{mOsm} / \mathrm{kg})$ at the time of diagnosis [7-9]. Confirmation of CDI was based on its reversal with desmopressin (DDAVP) and vasopressin infusion therapy $[9,10]$.

The SPSS version 15.0 was used for statistical analysis. Results were analysed with descriptive statistics.

Patients $(\mathrm{n}=341)$ who died in PICU between 2012 and 2016 were screened. In 37 of 341 deaths (10.8\%), the diagnosis of $\mathrm{BD}$ had been recorded. The male to female ratio was $1.05,19$ male $(51.4 \%)$ and 18 female patients (48.6\%). The median age of the patients was 6 (IQR: 2-9) years. The primary insult causing brain death was post-cardiorespiratory arrest in $9(24.3 \%)$, head trauma in $8(21.6 \%)$, and drowning in $4(10.8 \%)$ (Table 1).

\section{RESULTS}

The median duration of hospitalisation was 12 days (IQR: 9-17). The median duration of time between hos-

\section{Table1. Demographics of the Patients Who Were Declared Brain Dead}

\begin{tabular}{lc} 
Variable & $\begin{array}{c}\text { n(\%) or Median } \\
\text { [Interquartile Range] }\end{array}$ \\
\hline Number (\% of deaths) & $37(10.8 \%)$ \\
Age (yr) & $6[2,9]$ \\
Gender (male) & $19(51.4 \%)$ \\
Primaryhospitaladmissiondiagnosis & \\
Post-cardiorespiratory arrest & $9(24.3 \%)$ \\
Head trauma & $8(21.6 \%)$ \\
Drowning & $4(10.8 \%)$ \\
Sepsis and multiorgan dysfunction & $2(5.4 \%)$ \\
Status epilepticus & $2(5.4 \%)$ \\
Brain tumour & $2(5.4 \%)$ \\
Other etiologies & $4(10.8 \%)$ \\
Organ donors & $6(16.2 \%)$
\end{tabular}


pitalisation and the start of $\mathrm{BD}$ evaluation was seven days (IQR:5-10.5), and the median duration of brain death evaluation was three days (IQR:2-7). In the current study, we first confirmed $\mathrm{BD}$ in patients by clinical criteria then performed a confirmatory ancillary test.

In all patients, transcranial Doppler ultrasound ( TCD) was utilised as an additional test. The examination was repeated daily until BD was confirmed ultrasonographically. In four patients for whom initial TCD examination failed to establish $\mathrm{BD}$, a second TCD examination was applied. After that, clinical BD that showed confirmation of TCD signs was seen in three patients. The remaining patient underwent five TCD examinations that showed confirmatory TCD signs of BD.

In 33 (89\%) patients, CDI was determined to have developed at or near the time brain death was confirmed. All patients diagnosed with CDI received DDAVP and polyuria was reversed. All four patients not diagnosed with CDI had acute renal failure, and renal replacement treatment was applied. Laboratory test and admission diagnosis of these four patients are given in Table 2.

All the families were approached regarding organ donation, and consent was obtained from seven ( 18.9\%). One had mutliorgan dysfunction syndrome, and the other six (16.2\%) were medically suitable and were accepted as organ donors.From six donors, ten kidneys, five livers and two heart were transplanted successfully.Characteristics of these six donors are given in Table 3.

\section{Discussion}

BD accounted for $10.8 \%$ of the deaths in this study. Different pediatric studies have found different rates between $11.6 \%$ and $37 \%$ [11-13]. These differences may be due to the type of ICU, e.g., trauma referral centres, local practice differences such as the emphasis on organ donation, comfort with the determination of $\mathrm{BD}$, the year of study, and patient demographics.

In the current study, the primary diagnosis leading to brain death was post-cardiorespiratory arrest. Deprivation of oxygen and nutrient delivery to brain tissue is the most common underlying pathophysiology irrespective of the proximate cause leading to $\mathrm{BD}$. Hypoxic-ischemic brain injury and the resulting oedema evolve, and may ultimately progress to $\mathrm{BD}$ after brainstem function completely disappears usually in a rostrocaudal direction, from the mesencephalon to the medulla oblongata. In most studies, the most common finding in children is reported to be traumatic brain injury $[12,4,13,14]$. Some studies have found a high prevalence of infectious diseases [15] and cerebrovascular accidents [11].

In a recent meta-analysis of 32 studies, concerning 1878 patients with $\mathrm{BD}$, ranging in age from 2 months to 89 years, 925 (49\%) were reported to have CDI [6]. The five studies that have published exclusively on pediatric patients, collectively indicated that 145 (52\%) of 279 patients had CDI $[3,4,9,16,17]$. The data of the current study suggest that the prevalence of CDI in our pediatric $\mathrm{BD}$ population is higher (89\%) than other reports

Table 2. Laboratory Tests and Diagnosis of Patients Without CDI

\begin{tabular}{ccccc} 
& Age & BUN (mg/dL) & Creatinin (mg/dL) & Diagnosis \\
Patient 1 & 5 months & 46,6 & 2,7 & Post-CRA \\
Patient 2 & 1 years & 98 & 2,5 & Head trauma \\
Patient 3 & 8 years & 178 & 6,5 & Post-CRA \\
Patient 4 & 9 years & 111 & 7,3 & Head trauma \\
\hline CRA:Cardiorespiratory arrest & & & &
\end{tabular}

Table 3. Characteristics of Donors

\begin{tabular}{cccc} 
& Age & Diagnosis & Transplanted organs \\
Donor 1 & 5 months & Post-CRA & Liver \\
Donor 2 & 3.5 years & Drowning & Liver,2 kidney \\
Donor 3 & 5 years & Head trauma & 2 kidney \\
Donor 4 & 6 years & Post-CRA & Heart,2 kidney \\
Donor 5 & 17 years & Acute hydrocephalus & Heart,liver,2 kidney \\
Donor 6 & 2 years & Head trauma & Heart,2 kidney \\
\hline
\end{tabular}


in literature. The four patients not diagnosed with CDI had acute renal failure, and renal replacement treatment was undertaken. Acute renal failure results in the diminution of the amount of fluid delivered to the distal tubule and hence would limit renal water excretion and prevent polyuria. Theoretically, this phenomenon may occur even in the absence of AVP, and CDI was not diagnosed in these patients. Excluding the patients with acute renal failure, in the current study population, CDI prevalence was $100 \%$.

Brain functions may continue in many BD brains[18]. For example, electroencephalographic (EEG) activity has been documented in more than $20 \%$ of brain death patients [19], cerebral blow has been recorded in more than $5 \%$ of brain death patients [20], and it has been stated that CDI does not occur in more than half of brain death patients [6]. The most straightforward explanation for the observation of normouria in patients with $\mathrm{BD}$ is that the hypothalamic osmoregulation system continues to function, in at least some patients. The various findings consistent with the preservation of hypothalamic control of the anterior pituitary corroborate this by suggesting preserved blood flow to the area, and suggest some hypothalamic-anterior pituitary function as well, albeit often in the presence of peripheral endocrine insufficiency[6]. Furthermore, anatomical considerations indicate that preserved hypothalamic osmoregulation may be expected in BD. The inferior hypophyseal arteries branch off the extradural segments of the internal carotids and thus are protected from increased intracranial pressure, and these arteries supply the posterior pituitary, the hypophysial stalk, and parts of the hypothalamus including the median eminence [21].

The diagnosis of $\mathrm{BD}$ is clinical. In some countries, e.g., United States, confirmation of BD at the bedside is sufficient [1]. However, other countries recommend examinations that confirm the absence of electrical and metabolic functions or of cerebral flow. EEG is the most widely used examination in several countries. Nevertheless, this examination cannot be easily performed in an intensive care unit, due to the possible interference of artefacts with electrical activity [1]. TCD has been validated as an ancillary test for brain death with specificity of $98 \%$ to $100 \%$ and sensitivity ranging from $88 \%$ to $99 \%$, and it is performed on middle and vertebral cerebral arteries [22,23]. In Turkey, the law requires an additional testing, together with an apnea test for diagnosis of $\mathrm{BD}$ in children. In our clinic, a TCD, as an additional test, was conducted on all patients with a clinical diagnosis of $\mathrm{BD}$. This TCD test was repeated until it was consistent with BD. Studies in literature, which have researched the frequency of CDI accompanying $\mathrm{BD}$, give no information related to how $\mathrm{BD}$ was diagnosed and which additional tests were applied. That the rate of CDI in cases diagnosed with BD was higher in the current study compared to reports in the literature may be explained by the fact that brain blood circulation may not be shown in all patientswith TCD.

In the current study, the consent rate for organ donation was $18.9 \%$, and the organs of $16.2 \%$ of potential donors proceeded to actual donation. In a Canadian study, the consent rate was 64\%[12]. Tsai et al., (2005) reported a consent rate of $63 \%$, although the organs of only $41 \%$ of potential donors proceeded to actual donation[24], while in the Canadian study, organs of $47 \%$ of donated were used [12]. In the current study, the major reason for a patient not being an organ donor candidate was the family's refusal, and this was the case in $30(81 \%)$ of the patients. In some studies, religion, primary language and place of residence have all been highly correlated with ethnic background, and affect the consent rate $[25,26]$.

Donation rates may increase with carefully planned public educational broadcasting campaigns. We believe that the more information given to a family regarding the medical condition of their child, the better prepared the family members will be to having a conversation about organ donation.

In conclusion, the persistence of cerebral blood flow after $\mathrm{BD}$ can be considered to result in normouria in at least some patients. Further studies are required to explain the normouria in $\mathrm{BD}$ patients.

\section{CONFLICT OF INTEREST}

None to declare.

\section{REFERENCES}

1. Wijdicks EF. Brain death worldwide: accepted fact but no global consensus in diagnostic criteria. Neurology. 2002;58:20-5.

2. Gündüz RC, Şahin Ş, Uysal-Yazıcı M, Ayar G, et al. Brain death and organ donation of children.Turk J Pediatr. 2014;56:597603.

3. Fackler JC, Troncoso JC, Gioia FR. Age-specific characteristics of brain death in children. Am J Dis Child. 1988;142:999-1003.

4. Staworn D, Lewison L, Marks J, Turner G, Levin D. Brain death 
16 The Journal of Critical Care Medicine 2018;4(1)

in pediatric intensive care unit patients: incidence, primary diagnosis, and the clinical occurrence of Turner's triad. Crit Care Med. 1994;22:1301-5.

5. Varelas PN, Rehman M, Abdelhak $\mathrm{T}$, et al. Single brain death examination is equivalent to dual brain death examinations. Neurocrit Care. 2011;15:547-53.

6. Nair-Collins M, Northrup J, Olcese J.HypothalamicPituitaryFunction in Brain Death: A Review.J Intensive Care Med. 2016;31:41-50

7. Wise-Faberowski L, Soriano SG, Ferrari L, et al: Perioperative management of diabetes insipidus in children. J Neurosurg Anesthesiol. 2004;16:220-25.

8. Mishra G, Chandrashekhar SR.Management of diabetes insipidus in children. Indian J Endocrinol Metab. 2011;15:S1807.

9. Alharfi IM, Stewart TC, Foster J, Morrison GC, Fraser DD. Central diabetes insipidus in pediatric severe traumatic brain injury.Pediatr Crit Care Med. 2013;14:203-9.

10. Bajpai A, Kabra M, Menon PS: Central diabetes insipidus: Clinical profile and factors indicating organic etiology in children. Indian Pediatr. 2008;45:463-8.

11. Lago PM, Piva J, Garcia PC, et al.Brain death: medical management in seven Brazilian pediatric intensive care units.J Pediatr (Rio J). 2007;83:133-40.

12. Joffe AR, Shemie SD, Farrell C, Hutchison J, McCarthy-Tamblyn L. Brain death in Canadian PICUs: demographics, timing, and irreversibility.Pediatr Crit Care Med. 2013;14:1-9.

13. Goh AY, Mok Q: Clinical course and determination of brainstem death in a children's hospital. Acta Paediatr. 2004; 93:47-52.

14. Gotay-Cruz F, Fernández-Sein A: Pediatric experience with brain death determination. P R Health SciJ. 2002;21:11-5.

15. Ruiz-García M, Gonzalez-Astiazarán A, Collado-Corona MA, et al: Brain death in children: Clinical, neurophysiological and radioisotopic angiography findings in 125 patients. Childs Nerv
Available online at: www.jccm.ro

Syst. 2000;16:40-5.

16. Outwater KM, Rockoff MA. Diabetes insipidus accompanying brain death in children. Neurology. 1984;34:1243-6.

17. Finfer S, Bohn D, Colpitts D, Cox P, Fleming F, Barker G. Intensive care management of paediatric organ donors and its effect on post-transplant organ function.Intensive Care Med. 1996;22:1424-32.

18. Joffe AR, Anton N: Brain death: Understanding of the conceptual basis by pediatric intensivists in Canada. Arch Pediatr Adolesc Med. 2006;160:747-52.

19. Paolin A, Manuali A, Di Paola F, et al. Reliability in diagnosis of brain death. Intensive Care Med. 1995;21:657-62.

20. Flowers WM Jr, Patel BR. Persistence of cerebral blood flow after brain death. South Med J. 2000;93:364-70.

21. Leclercq TA, Grisoli F. Arterial blood supply of the normal human pituitary gland. An anatomical study. J Neurosurg. 1983;58:678-81.

22. de Freitas GR, Andre C. Sensitivity of transcranial Doppler for confirming brain death: a prospective study of 270 cases. Acta Neurol Scand. 2006;113:426-32.

23. Wijdicks EFM. Brain death. New York, NY: Oxford University Press; 2011.

24. Tsai WH, Lee WT, Hung KL. Determination of brain death in children-a medical center experience. Acta Paediatr Taiwan. 2005; 46: 132-7.

25. Tsai E, Shemie SD, Cox PN, Furst S, McCarthy L, Hebert D. Organ donation in children: role of the pediatric intensive care unit. Pediatr Crit Care Med. 2000;1:156-60.

26. McQuay JE. Cross-cultural customs and beliefs related to health crises, death, and organ donation/ transplantation: a guide to assist health care professionals understand different responses and provide cross-cultural assistance. Crit Care Nurs Clin North Am. 1995; 7: 581-94. 